\title{
On the Riemann Theta Function of a Trigonal Curve and Solutions of the Boussinesq and KP Equations
}

\author{
V. B. MATVEEV and A. O. SMIRNOV $\star \star$ \\ II. Institut für Theoretische Physik, Universität Hamburg, Luruper Chaussee 149. \\ 2 Hamburg 50, West Germany
}

(Received. 9 January 1987)

\begin{abstract}
Recentiy, considerable progress has been made in understanding the nature of the algebrogeometrical superposition principles for the solutions of nonlinear completely integrable evolution equations, and mainly for the equations related to hyperelliptic Riemann surfaces. Here we find such a superposition formula for particular real solutions of the KP and Boussinesq equations related to the nonhyperelliptic curve $\omega^{4}=\left(\lambda-E_{1}\right)\left(\lambda-E_{2}\right)\left(\lambda-E_{3}\right)\left(\lambda-E_{4}\right)$. It is shown that the associated Riemann theta function may be decomposed into a sum containing two terms, each term being the product of three one-dimensional theta functions. The space and time variables of the KP and Boussinesq equations enter into the arguments of these one-dimensional theta functions in a linear way.
\end{abstract}

\section{Introduction}

Until recent times, simple examples of nonhyperelliptical solutions of the Boussinesq equation

$$
3 u_{y y}+\left(u_{x x x}+6 u u_{x}\right)_{x}=0,
$$

reducible to one-dimensional theta functions, were unknown. The curve $\Gamma$ :

$$
\omega^{4}=\left(\lambda-E_{1}\right)\left(\lambda-E_{2}\right)\left(\lambda-E_{3}\right)\left(\lambda-E_{4}\right), \quad \operatorname{Im} E_{k}=0,
$$

was discussed in [1] as an example of Krichever's reduction of the KP equation to a Boussinesq equation via Weierstrass points. But at that time only the Weierstrass points coinciding with branch points were explored, but the possibility of reducing associated three-dimensional theta functions to one-dimen sional theta functions was not discussed. Such a possibility arises from the existence of the conformal automorphism $\tau:(\omega, \lambda) \rightarrow(i \omega, \lambda)$ interchanging the sheets of the associated Riemann surface, realized as a four-sheeted covering of the complex $\lambda$-plane. The way to explore this automorphism for reducing three-dimensional Riemann theta functions goes back to the

* On leave from Leningrad State University and Leningrad Institute of Avation Instrumentation. Permanent address after January 1987: Department of Physics, Division of Mathematical Physics of Leningrad University, 198904, Pervomayaskaya 100, Leningrad, U.S.S.R.

$\star \star$ Pemanent address: Department of Mathematics of the Leningrad Institute of Aviation Instrumentathon, 190000, Gertzena 67, Leningrad, U.S.S.R. 
methods given in [2,3] - see also [4] - although application of the matrix version of Appel's theorem used below simplifies the calculations. As a main result we obtain a family of the genus three solutions of the KP and Boussinesq equations expressed by means of elliptic theta functions.

\section{The Algebrogeometrical Solutions of the $\mathrm{KP}$ and Boussinesq Equations}

The formula

$$
u(x, y, t)=2 \frac{\partial^{2}}{\partial x^{2}} \ln \theta(x \mathrm{U}+y \mathbf{V}+t \mathbf{W}-\mathbf{I} \mid B)+C
$$

describes the solutions of the Kadomtsev-Petviashvili (KP) equation

$$
3 u_{y y}+\left(u_{x x x}+6 u u_{x}-4 u_{t}\right)_{x}=0,
$$

generated by an arbitrary compact Riemann surface $\Gamma$ [5]. In Equation (2) $B$ means the matrix of $b$ periods of the surface $\Gamma$ in some canonical basis of $H_{1}(\Gamma) . \theta$ is a $g$-dimensional theta function defined by the formula

$$
\begin{aligned}
& \theta(\mathbf{p} \mid B)=\theta\left[\begin{array}{l}
0 \\
0
\end{array}\right](\mathbf{p} \mid B), \\
& \theta\left[\begin{array}{l}
\alpha \\
\beta
\end{array}\right](\mathbf{p}, B)=\sum_{\mathbf{m} \in Z^{g}} \exp \{\pi i\langle B(m+\alpha), m+x\rangle+2 \pi i\langle m+\alpha, p+\beta\rangle\},
\end{aligned}
$$

where $g$ is the genus of the Riemann surface $\Gamma . \mathrm{U}, \mathbf{V}, \mathbf{W}$ are the vectors of $b$-periods of some normalized Abelian integrals of the second kind with the poles at the marked point $P_{0} \in \Gamma$. In the case of the hyperelliptic curve, $P_{0}$ may coincide with one of the branch points. In this case, vector $\mathbf{V}$ turns out to be equal to zero and formula (2) reduces to the solution of the $\mathrm{KdV}$ equation, given by the Its-Matveev formula [1, 6-8]. If $\Gamma$ is some trigonal curve, i.e., there exists a meromorphic function with a unique pole at the point $P_{0}$ of the order 3, it turns out that $W=0$. In this case, the KP solution (2) is independent on $t$ and satisfies the nonlinear Boussinesq equation (1). Trigonal curve 3 of the genus $g=3$ cannot be hyperelliptic. The curve $\Gamma$ considered in this Letter is nonhyperelliptic of the genus 3 . It is of the nondividing type. Its branch points are $P_{j}=\left(0, E_{j}\right)$.

Let $P_{0}$ coincide with one of the branch points. It is possible to construct a local parameter $K(P), P \in \Gamma$ in such a way that under the action of the antiholomorphic involution $\tau:(\omega, \lambda) \rightarrow(\bar{\omega}, \bar{\lambda})$, it transforms to $\bar{k}$ or $-\bar{k}$. Let $\eta_{j}(m)$ be the path with the starting point at $\left(0, E_{l}\right)$ and ending at $\left(0, E_{j}\right)$, $\arg \omega(\lambda)=(\pi m) / 4$ along the path. The canonical basis of the curve $\Gamma$ may now be defined by

$$
a_{1}=\gamma_{34}(1)+\gamma_{43}(-1), \quad a_{2}=\gamma_{34}(-3)+\gamma_{43}(3),
$$




$$
\begin{aligned}
& a_{3}=\gamma_{23}(2)+\gamma_{32}(-2), \\
& b_{1}=\gamma_{34}(3)+\gamma_{43}(1)+\gamma_{23}(4)+\gamma_{32}(-2), \\
& b_{2}=\gamma_{34}(-1)+\gamma_{43}(-3)+\gamma_{23}(0)+\gamma_{23}(-2), \\
& b_{3}=\gamma_{21}(1)+\gamma_{21}(3) .
\end{aligned}
$$

Let I satisfy the condition $\operatorname{Re} l_{j}=\frac{1}{2} \operatorname{Re} B_{j r}$.

Under the assumptions of this point, we have the following main theorem.

THEOREM 1. The solution of the Boussinesq equation defined by the curve $\Gamma$ is real-valued and may be reduced to the foliowing form:

$$
\begin{aligned}
u= & 2 \partial_{x}^{2} \ln \left\{\theta\left[\begin{array}{c}
0 \\
\frac{1}{2}
\end{array}\right]\left(p_{1} \mid B_{1}\right) \theta\left(p_{2} \mid B_{2}\right) \theta\left(p_{3} \mid B_{2}\right)+\right. \\
& \left.+\theta\left[\begin{array}{l}
1 \\
2 \\
\frac{1}{2}
\end{array}\right]\left(p_{1} \mid B_{1}\right) \theta\left[\begin{array}{l}
1 \\
2 \\
\frac{1}{2}
\end{array}\right]\left(p_{2} \mid B_{2}\right) \theta\left[\begin{array}{c}
\frac{1}{2} \\
\frac{1}{2}
\end{array}\right]\left(p_{3} \mid B_{2}\right)\right\}+C-2 \pi i \frac{U_{3}^{2}}{B_{33}}, \\
B_{1}= & 4 B_{12}+B_{33}-B_{33}^{-1}+2, \quad B_{2}=-B_{33}^{-1}, \\
p_{1}= & x\left(U_{1}+U_{2}\right)+y\left(V_{1}+V_{2}\right)+l_{1}+l_{2}, \\
p_{2}= & x\left(U_{1}-U_{2}-U_{3}\right)+y\left(V_{1}-V_{2}-V_{3}\right)+l_{1}-l_{2}-l_{3}-\frac{1}{2}, \\
p_{3}= & B_{33}^{-1}\left(x U_{3}+y V_{3}+l_{3}\right) .
\end{aligned}
$$

\section{Proof of the Main Theorem}

The reality of the solution ( 7 ) of the Boussinesq equation follows directly from the recent results of Dubrovin [9]. The crucial step of the proof of the formula (7) is to demonstrate the associated decomposition formula for the Riemann theta function of the curve $\Gamma$. To produce such a decomposition formula, we start by specifying the structure of the matrix $B$ taking into account the symmetry properties of the curve $\Gamma$

LEMMA 1. Let $\tau_{1}$, be a holomorphic automorphism of some compact Riemann surface $\Gamma$ of the genus 3 which acts on the basis of $H_{1}(\Gamma)$ in the following way:

$$
\tau_{1} \mathbf{a}=M \mathbf{a}, \quad \tau_{1} \mathbf{b}=\left(M^{T}\right)^{-1} \mathbf{b}+L \mathbf{a} .
$$

Then the $B$ matrix satisfies the relation

$$
B=M^{T} B M-M^{T} L .
$$

Proof of Lemma 1 follows directly from the general laws - see [4] - of transformation of the $B$ matrix under the change of the canonical hasis of $H_{1}(\Gamma)$. 
Under the conditions of Lemma 1, we can find a matrix $T, T_{i k} \in Z$, in such a way that the conditions

$$
\begin{aligned}
& B^{\prime}=G B^{\prime} G, \quad G=T^{-1} M T, \\
& B^{\prime}=T^{T}(B-A) T,
\end{aligned}
$$

where $A$ is a symmetric matrix with rational matrix elements satisfying the same relation (10) as the $B$ matrix, and leads to a block diagonal structure of the matrix $B^{\prime}$.

Let condition (11) be satisfied and the elements of the matrix $A^{\prime}-D$ defined by $A^{\prime}=T^{T} A T, D=\operatorname{diag}\left[A_{i i}^{\prime}\right]$, are entries.

Now it is easy to prove the following theorem.

THEOREM 2. Under the condition of this point, a g-dimensional theta function generated by the $B$ matrix may be represented in the form:

$\theta\left[\begin{array}{l}\alpha \\ \beta\end{array}\right](p \mid B)=\sum_{s \in Z^{x^{\prime}(T)}} \exp \left\{-\pi i\left\langle\left(A^{\prime}-D\right) \alpha(s), \alpha(s)\right\rangle\right\} \theta\left[\begin{array}{l}\alpha(s) \\ \beta(s)\end{array}\right]\left(T^{T} p \mid B^{\prime}+D\right)$.

Summing over $\mathcal{Z}^{g}(T)$ means that $s \in Z^{g}$ runs over all the vectors constrained by the inequality $0 \leqslant\left(T^{-1} s\right)_{j}<1$.

$$
\alpha(s)=T^{-1}(s+\alpha), \quad \beta=\left(A^{\prime}-D\right) \alpha(s)+T^{\top} \beta .
$$

Taking into account that $B^{\prime}$ is a block structure and $D$ is a diagonal matrix, it is evident that

$$
\theta\left[\begin{array}{l}
\alpha(s) \\
\beta(s)
\end{array}\right]\left(T^{T} p \mid B^{\prime}+D\right)
$$

may be represented in the form of a product of lower-dimension theta functions.

Proof of Theorem 2 consists simply of going from summing over $m \in Z^{g}$ to summing over $n$ and $s$ related to $m$ by the equality $m=T n+s$. The number of terms in the sum taken over $Z^{g}(T)$ is equal to $|\operatorname{det} T|$ because $\operatorname{det} T$ is the Jacobian determinant of the transformation from the $m$-lattice to the $n$-lattice.

Theorem 2 is related to Theorem 1 in the following way. Let us introduce a new canonical basis in $H_{1}(\Gamma)$ :

$$
\tilde{a}_{3}=-b_{3}, \quad \tilde{b}_{3}=a_{3}, \quad x_{k}=a_{k}, \quad \tilde{b}_{k}=b_{k}, \quad k=1,2 .
$$

Now the holomorphic automorphism appearing in Lemma 1 may be realized so that $\tau_{1}: E_{k} \rightarrow E_{S-k}, \omega \rightarrow i \omega$. Then we find that the matrices $A$ and $T$ may be chosen in the form

$$
r=\left[\begin{array}{rrr}
1 & 1 & 0 \\
-1 & -1 & 0 \\
-1 & 0 & -1
\end{array}\right], \quad A=\left[\begin{array}{lll}
0 & 0 & 1 \\
0 & 0 & 0 \\
1 & 0 & 1
\end{array}\right]
$$


Now a direct application of Theorem 2 leads to the representation (7) for the solutions of the Boussinesq nonlinear equation.

REMARK. If the point $P_{0}$ does not coincide with one of the Weierstrass points, from decomposition (13) we get the solutions of the KP equation represented in terms of one-dimensional theta functions.

\section{Weierstrass Points of the Curve $\Gamma$, Which are not Branch Points}

Weierstrass points of the curve $\Gamma$ were studied in [10] where a complete description of the Weierstrass points of the genus 3 curves was presented. However, the variety of Weicrstrass points of the curve $\Gamma$ pointed out in [10] seems to be wrong because it is not invariant with respect to the action of some conformal automorphisms. That is why we recalculate the $W$-points of the curve $\Gamma$. This calculation may be performed in the standard way by looking at the behaviour of the Wronskian of normalized Abelian differentials. Here we give only the final answer, formulated for the curve

$$
y^{4}=x^{4}-\left(m^{2}+1\right) x^{2} z^{2}+m^{2} z^{4} .
$$

This curve, given by the polynomial equation (15), where $x, y, z$ are homogenous coordinates, is birationally equivalent to the curve $\Gamma$. So it is sufficient to describe all Weierstrass points in this realization of $\Gamma$. These are two possibilities: $m^{2}=-1$, $m^{2} \neq-1$ which are essentially different in the study of $W$-points. First, let $m^{2}=1$. In this case, the number of Weierstrass points is 12 . Their positions are

$$
\left(0, i^{k}, \exp (i \pi / 4)\right),\left(i^{k}, 0,1\right),\left(i^{k}, 1,0\right), \quad k=1,2,3,4 \text {. }
$$

The meromorphic functions with a unique singularity pole of the third order at these points are

$$
x \cdot\left\{y \mathrm{e}^{(\pi) / 4}-i^{k} z\right\}^{-1}, \quad y\left(x-i^{k} z\right)^{-1}, \quad z\left(x-i^{k} y\right)^{-1},
$$

respectively.

In the case $m^{2} \neq-1$, we have 20 Weierstrass points. These $W$-points may be divided in two groups. The first group contains all the branch points and meromorphic functions with a third-order pole as a unique singularity that may be constructed as above. The sccond group contains 16 points with the positions $\left(x_{k}, y_{i k}, 1\right)$ defined from the system

$$
\begin{aligned}
& y_{i k}^{4}=x_{k}^{4}-\left(m^{2}+1\right) x_{k}^{2}+m^{2}, \\
& 2\left(m^{2}+1\right) x_{k}^{4}+\left(m^{4}-10 m^{2}+1\right) x_{k}^{2}+2 m^{2}\left(m^{2}+1\right)=0 .
\end{aligned}
$$

The related meromorphic functions are of the form $\left(x-\tilde{x}_{k}\right)\left(y-y_{k k}-y_{k k}^{\prime}\left(x-x_{k}\right)^{-1}\right)$, stays for the differentiation on $x$ and

$$
\tilde{x}_{k}=x_{k}+4\left(x_{k}-y_{i k}\left(y_{i k}^{\prime}\right)^{3}\right)\left[\left(y_{t k}^{\prime}\right)^{4}-1\right]^{1} \text {. }
$$




\section{The Reduction of the Basis Abelian Integrals of the First Kind to the Elliptic Integrals}

$$
\int \frac{d \lambda}{\omega^{3}} \text { and } \int \frac{\lambda d \lambda}{\omega^{3}}
$$

may be reduced to the linear combination of the integrals $I_{1,2}$ :

$$
I_{1}=\int \frac{\mu \mathrm{d} \mu}{\left[\left(\mu^{2}-1\right)\left(\mu^{2}-m^{2}\right)\right]^{3 / 4}}, \quad I_{2}=\int \overline{\left[\left(\mu^{2}-1\right)\right.} \frac{\mathrm{d} \mu}{\left.\left(\mu^{2}-m^{2}\right)\right]^{3 / 4}}
$$

by a fraction-linear transformation.

$I_{2}$ may be reduced to $I_{1}$ by a change of variables $\mu=m v^{-1}$.

$I_{1}$ may be reduced by the transformation

$$
\left.y=\frac{2}{\left(1-m^{2}\right)} \sqrt{\left(\mu^{2}\right.}-1\right)\left(\mu^{\overline{2}}-m^{2}\right)
$$

to the elliptic integral

$$
I_{1}=\frac{(i)^{k}}{\sqrt{2\left(1-m^{2}\right)}} \int \frac{\mathrm{d} y}{\sqrt{y\left(1+y^{2}\right)}}
$$

where the choice of $k$ depends on the path of integration. The third basis integral $\int \mathrm{d} \lambda / \omega^{2}$ is just elliptic and needs no further reduction.

\section{Concluding Remarks}

This Letter is part of a program [2-4] of studies on theta functions of Riemann surfaces with nontrivial automorphisms and their applications to soliton equations. Such surfaces and their theta functions are also of interest for the recent developments of quantum string theories. Such surfaces appear particularly in the description of the interactions on orbifolds involving the emission of twisted states [11]. For example, the famous Klein curve with a simple group of birational automorphisms of the extremal order 168 (see [2] for a study of its $B$ matrix and particular properties of its theta function) is also encountered in the classification of orbifolds with SU(3) holonomy [12].

\section{Acknowledgements}

V.B.M. is grateful to Professors J. Bartels and H. Lehmann for their kind hospitality at the II. Institute of Theoretical Physics of Hamburg University where this work was finished. 


\section{References}

1. Matvecv, V. B., Abelian functıons and solitons, Preprint No. 373, Unversity of Wroclaw, 1976, p. 1.98.

2. Babich, M. B., Bobenko, A. I., and Matveev, V. B., DAN SSSR 272:1, 13.17 (1983).

3. Babich, M. V., Bobenko, A. 1., and Matveev, V. B., Izv. Acad. Nauk. ser. math. 49:13, 511-529.

4. Belokolos, E. D., Bobenko, A. I., Matveev, V. B.. and Enolskı, V. Z., Uspehi Mat. Nauk 41:2, 3-42 (1986).

5. Krichever, I. M., Functional Analysis and Applications 11:1, 15.31 (1977).

b. Its, A. R. and Matvecv, V. B.. Theor. Math. Phys. 23:1, 51-67 (1975).

7. Matveev, V. B., Uspehi Math, Nauk 30:6, 201-203 (1975).

8 Dubrown, B. A., Matvee, V. B., and Navikov, S P., Uspehi Math. Nauk 31:1, 55-136 (1976).

9. Dubrovin, B. A., in Advanced Problems in Mathemancs, Vol 23, VINITI, 1983, p. 33-78.

10. Kuribayashi, A. and Komya, K., On Wererstrass points and automorphisms of curves of genus three, Lecture Noles in Malh. 732, 1979, pp. 252-299.

11. Hamıdi, S. and Vafa, C. Nucl. Phys. B279, 465-513 (1987)

12 Dixon, L., Harvey, J., Vafa, C., and Witten, E., Nucl. Phy. B261, 678 (1985); thid. B274, 285 (1986) 